\title{
Weight Decision Scheme based on Slot-Count in Gen-2 Q-Algorithm
}

\author{
In-Taek Lim, Member, KIMICS
}

\begin{abstract}
In the Gen-2 Q-algorithm, the values of weight $C$, which is the parameter for incrementing or decrementing the slot-count size, are not optimized in the standard. However, the standard suggests that the reader uses small values of $C$ when the slot-count is large and larger values of $C$ when the slot-count is small. In this case, if the reader selects an inappropriate weight, there are a lot of empty or collided slots. As a result, the performance will be declined because the frame size does not converge to the optimal point quickly during the query round. In this paper, we propose a scheme to select the weight based on the slot-count size of current query round. Through various computer simulations, it is demonstrated that the proposed scheme achieves more stable performances than Gen-2 Q-algorithm.
\end{abstract}

Index Terms-RFID, Slot-count, EPCglobal Class-1 Gen2, Q-algorithm, Weight

\section{INTRODUCTION}

RECENTLY, the radio frequency identification (RFID) system attracts a lot of attention due to its automatic identification capability through RF communication. An RFID system consists of a reader and one or more tags with unique identifiers. The reader is a device, which can read and write tags by issuing RF signals to interrogate the identifier of attached tags. A tag is used to identify an object and is located in the object itself. In RFID system, tag identification is performed by the reader's query to a tag and then the tag's backscattering of its identifier as a response. However, if there are multiple tags within the identification range of reader, some of them might respond simultaneously and leads to collisions which decrease the performance. Therefore, the system requires a multiple-access scheme that allows the reader to read data from the individual tags. A technical scheme that handles multiple-access is called an anti-collision algorithm [1][2].

There are two types of anti-collision algorithms in RFID systems: probabilistic and deterministic algorithms. EPCglobal Class-1 Gen-2 and ISO/IEC 18000-6 Type C use the probabilistic algorithm as the standard [3][4]. The probabilistic algorithms are based on ALOHA-like protocol that provides slots for tags to send their data. Almost all the probabilistic algorithms use framed slot

\footnotetext{
Manuscript received March 7, 2011; revised April 2, 2011; accepted April 15, 2011.

In-Taek Lim is with the Department of Embedded IT, Pusan University of Foreign Studies, Busan, 608-738, Korea (Email: itlim@pufs.ac.)
}

ALOHA (FSA), which has been advanced in function by adding slotting and framing on ALOHA. The tags send their identifiers at a randomly selected slot. When collisions occur, the tags that are involved in a collision retransmit their identifiers in the next query round. The probabilistic algorithms may have limitations on the completeness of tag identification because there is still a probability of failing to be identified in a limited time period. The deterministic algorithms are used in EPCglobal Class-0 and ISO/IEC 18000-6 Type B, which uses binary tree-walking scheme [5].

EPCglobal Class-1 Generation-2 standard proposed Qalgorithm to determine the slot-count for the next query round [3]. The Q-algorithm determines the slot-count without conducting the tag number estimation scheme. Therefore, it wastes less computational cost and is simpler than other FSA algorithms. In Q-algorithm, the slot-count size may be incremented or decremented according to the status of reply slot. When the result of tag's reply in a slot is idle, the reader subtracts a weight $C$ from the slot-count. When a collision occurs, a weight $C$ is added to the slotcount. The standard did not specify the optimal weight $C$ value and remained it as an implementation-specific value. However, the standard suggests that the reader typically uses small values of $C$ when the slot-count is large and larger values of $C$ when the slot-count is small. If an inappropriate weight is selected, there may be a lot of idle or collided slots. As a result, the frame size will not converge to the optimal point quickly during the query round. This will reduce the identification speed and slot efficiency. Therefore, this paper proposes a scheme to decide the weight based on the slot-count size and status of tag's reply.

This paper is organized as follows. In Section II, we describe Gen-2 anti-collision algorithm and Q-algorithm. In Section III, we describe the problems of Q-algorithm and present the proposed scheme. Section IV shows the simulation results, and Section V concludes the paper.

\section{RELATED WORK}

\section{A. Gen-2 anti-collision algorithm}

In the Gen-2 anti-collision algorithm, the reader begins a query round by transmitting a Query command. After issuing a Query command to initiate a query round, the reader transmits one or more QueryRep commands to detect each slot during a query round. Fig.1 shows the simplified tag state transition diagram of Gen-2 anti- 
collision algorithm. Upon entering an energizing RF field, a tag that is not killed enters Ready state and remains in the Ready state until it receives a Query command. When tags receive a Query command, they draw a Q-bit number from their random number generator, load this number into their slot counter, and transition to the Arbitrate state if the number is nonzero, or to the Reply state if the number is zero.

A tag in the Arbitrate state decrements its slot counter every time it receives a QueryRep command, and it transitions to the Reply state and backscatters an RN16 when its slot counter reaches zero. If it receives a QueryAdjust command, it adjusts its Q value according to UpDn field of QueryAdjust command, selects a new random number, and loads this number into its slot counter. A tag that reinitialized its slot counter remains in the Arbitrate state if the slot counter is nonzero, or transitions to the Reply state.

Upon entering the Reply state, a tag backscatters an RN16. If the tag receives a valid acknowledgement, it transmits its PC, EPC, and $\mathrm{CRC}-16$, and enters the Acknowledged state. If the tag fails to receive an ACK command within the specified time, or receives an invalid ACK or an erroneous RN16, it returns to the Arbitrate state. After acknowledging a tag, a reader can choose to access it.

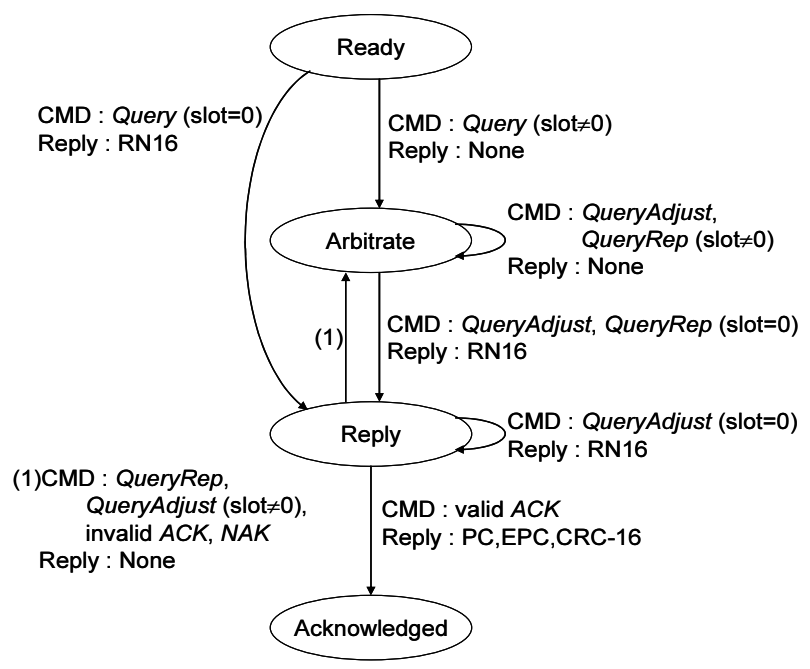

Fig.1. Tag state transition diagram.

\section{B. Gen-2 Q-algorithm}

In EPCglobal Class-1 Gen-2 RFID system, the Qalgorithm has been proposed to determine the slot-count in the next query round. Q-algorithm basically calculates the slot-count parameter $Q$ based on the result of tag's reply in a slot. The slot status is classified into three categories: success, collision, and empty slot.

Fig.2 shows an algorithm that the reader might use for setting the slot-count parameter $Q$ in a query round. In the figure, $Q_{f p}$ is a floating-point representation of $Q$. As shown in the figure, the reader updates $Q_{f p}$ in accordance with the slot status at every slot. When a collision occurs, it adds the weight $C$ value to the previous $Q_{f p}$, because it means the slot-count is smaller than the number of tags. If the result of tag's reply in a slot is idle, which means that there are no tag responses in the slot, the reader subtracts the weight $C$ value from the previous $Q_{f p}$, because the slot-count is larger than the ideal one. When a new query round begins, the reader rounds $Q_{f p}$ to an integer value $Q$ in the Query command. Typical values for the weight $C$ are $0.1<C<0.5$. EPCglobal Class- 1 Gen-2 standard suggests that the reader typically uses small values of $C$ when $Q$ is large and large values of $C$ when $Q$ is small.

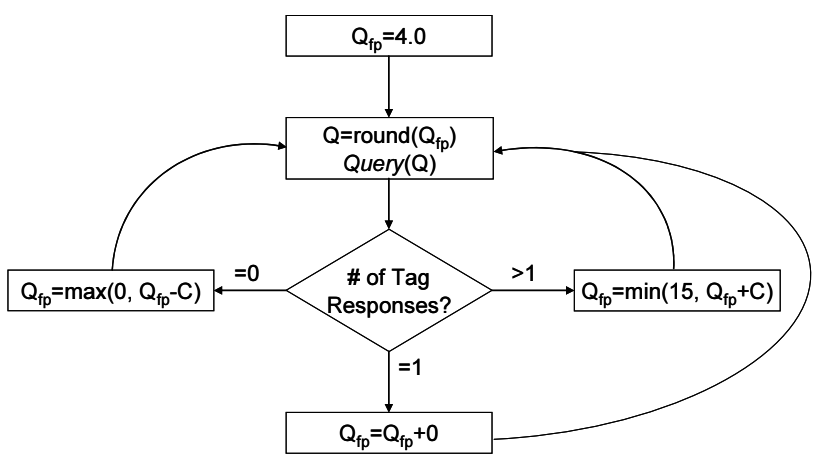

Fig.2. Gen-2 Q-algorithm.

\section{PROPOSED SCHEME}

\section{A. Research motivations}

In the Q-algorithm of Gen-2 RFID system, the weight $C$ values are not optimized. Therefore, we analyze how the performances of Gen-2 anti-collision algorithm are influenced by the weight depending on the number of tags within the identification range of reader.

The performance analysis was done by the computer simulations. The simulation parameters are same as the reference [6]. We assume that the Query commands will be transmitted as follows:

1) When there is a successful reply, the reader transmits QueryRep command.

2) When there is a collided reply or no reply, the reader sends QueryAdjust command if the slotcount gets changed or QueryRep command if the slot-count has no change.

Fig.3 and Fig.4 illustrate the identification speed and efficiency according to the weight $C$, respectively. The identification speed is defined as the number of tags identified in a second and the efficiency as the number of tags identified in a slot. As shown in the figures, when the number of tags is small, the identification speed and efficiency fluctuate severely. Also, the performances decrease when the weight $C$ is getting large. When the weight is large or the number of tags is small, the slotcount size will vary frequently. As a result, the slot-count does not converge to the optimal point quickly. Therefore, it seems that the weight has to be adjusted according to the slot-count size of current query round. 


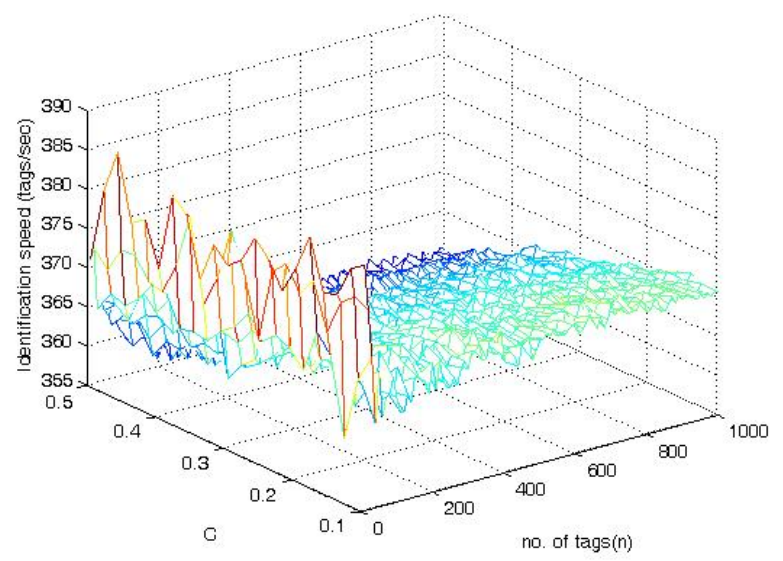

Fig.3. Identification speed according to weight $C$.

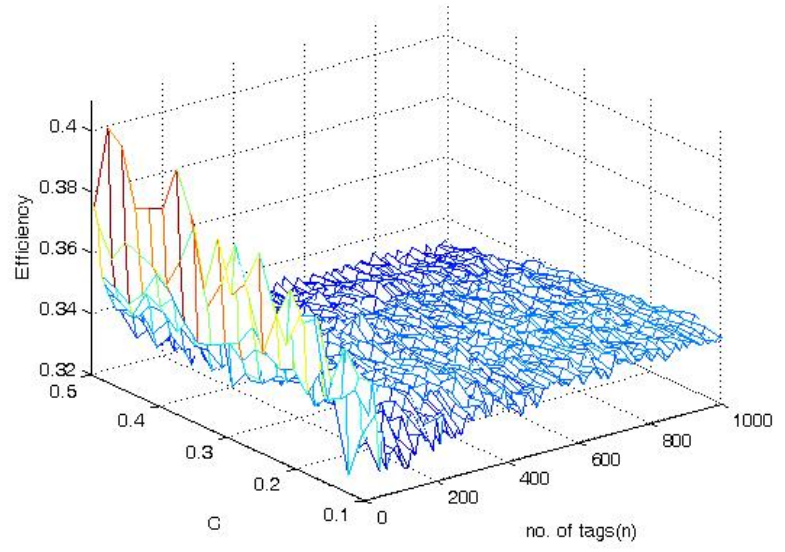

Fig.4. Efficiency according to weight $C$.

\section{B. Weight decision scheme}

The weight decision scheme to be explained later can be represented as a mathematical relation between the weight $C$ and slot-count of current query round. For deriving the mathematical relation, we let $N_{c}$ be the number of collision slots in the current query round. In FSA algorithm, the number of tags that are involved in collisions is $2.4 N_{c}$ when $N_{c}$ slots have collisions [6].

In the Gen-2 Q-algorithm, when the result of tag reply in a slot is collision, the reader adds the weight $C$ value to the slot-count. If $N_{c}$ slots are collisions, the slot-count size can be expressed as following:

$$
Q_{f p}=Q_{f p}+N_{c} C
$$

The optimal frame size for maximizing the performance of FSA algorithm can be taken with the analytical method. As a result of performance evaluation of FSA algorithm through analytical method, the optimal frame size is same as the number of tags within the identification range of reader [7]. The reference [6] showed that the number of tags involved in collisions is $2.4 N_{c}$ in the current query round when $N_{c}$ slots collide. Therefore, the number of slots $\left(N_{1}\right)$, which are needed in the next query round for maximizing the performances, is as follows:

$$
N_{1}=2^{Q_{f p}}+2.4 N_{c}
$$

On the other hand, the reader adds the weight to the slot-count value when collisions occur. After the reader updates the slot-count value due to $N_{c}$ collision slots, the number of slots $\left(N_{2}\right)$ in the new query round can be expressed as follows:

$$
N_{2}=2^{\left(Q_{f p}+N_{c} C\right)}
$$

The number of slots for obtaining the optimal performance $\left(N_{1}\right)$ and the number of slots in the new query round due to the updated slot-count value $\left(N_{2}\right)$ should be same. In the Gen-2 Q-algorithm, the reader updates the slot-count value whenever a collision occurs. Thus, $N_{c}$ in Eq.(2) and Eq.(3) becomes 1. From Eq.(2) and Eq.(3), we can express the weight $C$ as a mathematical relation of slot-count as following:

$$
C=\log _{2}\left(2^{Q_{f p}}+2.4\right)-Q_{f p}
$$

\section{SIMULATION RESULTS}

In this paper, we evaluate the performance for the proposed scheme through the computer simulations. The system parameters for computer simulations are same as the reference [6]. And it is assumed that the rules for transmitting Query command are same as Section III. We compare the proposed scheme with the original Qalgorithm. In the simulations, it is assumed that the initial slot-count value for both algorithms is 4.0. And we assume that the weight $C$ is 5.0 in the case of Gen-2 algorithm. All the results of simulation are averaged after iterating 100 times.

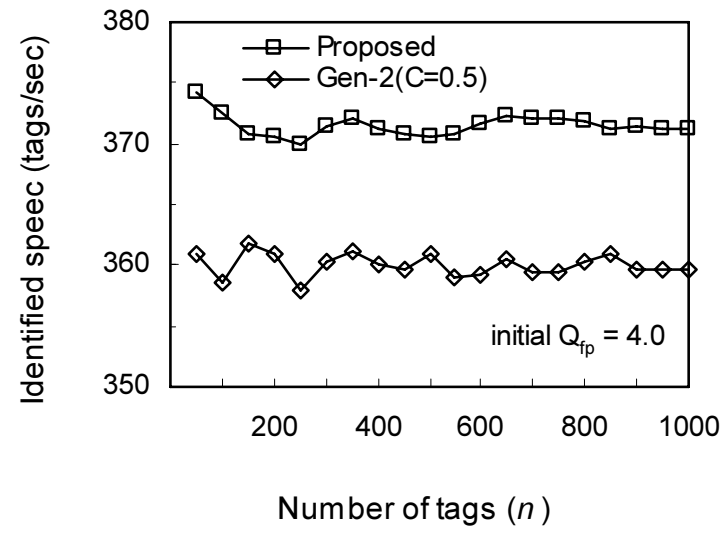

Fig.5. Identification speed (large number of tags).

Fig.5 and Fig.6 show the identification speed according to the number of tags within the identification range of reader. If there is large number of tags, the proposed scheme and Qen-2 algorithm can identify 371 tags and 360 tags for a second as shown in Fig.5, respectively. Thus, the proposed scheme can identify about $3.1 \%$ more tags than Gen-2 algorithm. On the other hand, as shown in 
Fig.6, the proposed scheme outperforms the Gen-2 algorithm by around $2.5 \%$ when the number of tags is less than 200 .

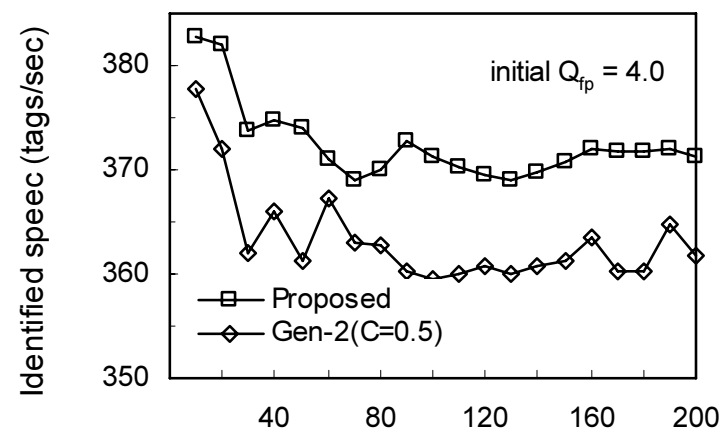

Number of tags $(n)$

Fig.6. Identification speed (small number of tags).

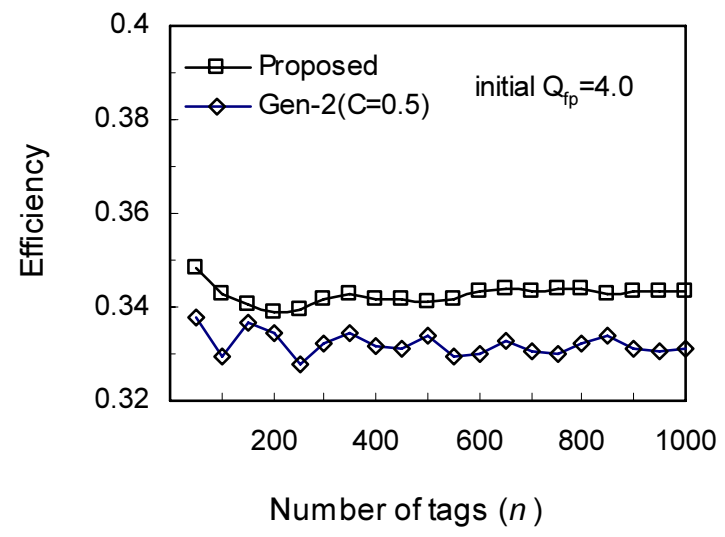

Fig.7. Slot efficiency (large number of tags).

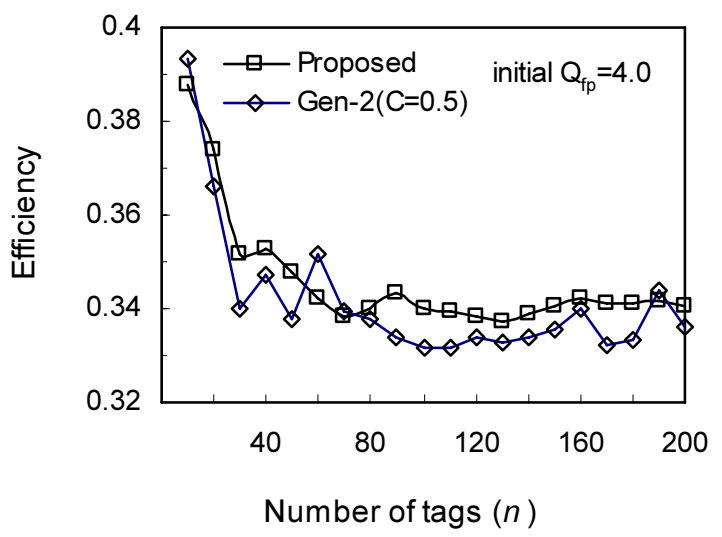

Fig.8. Slot efficiency (small number of tags).

Fig. 7 and Fig. 8 show the slot efficiencies in the case that the number of tags is large and small, respectively. When the number of tags is large, the slot efficiencies for the proposed scheme and Gen-2 algorithm are $34.3 \%$ and $33 \%$, respectively. As a result, the proposed scheme outperforms Gen-2 algorithm as shown in Fig.7. On the other hand, when the number of tags is small, there are no significant differences between the proposed scheme and Gen-2 algorithm. However, Fig. 8 shows that the proposed scheme is more stable than Gen-2 algorithm. This is because the proposed scheme adjusts the weight $C$ value based on the slot-count of current query round.

\section{CONCLUSIONS}

In EPCglobal Slass-1 Gen-2 RFID system, Q-algorithm is proposed to determine the slot-count for the next query round. In Q-algorithm, when the result of tag's reply is idle, the reader subtracts the weight $C$ from the slot-count. When a collision occurs, the reader adds the weight to the slot-count. However, the weight $C$ value is not optimized.

In this paper, we proposed a scheme to decide the weight $C$ value. In the proposed scheme, the weight was represented as a mathematical relation with the slot-count of current query round, and calculated by the reader for each slot. Throughout the simulation results, we clarified that the identification speed and efficiency of Gen-2 algorithm fluctuate severely when the number of tags is small. Also, the simulation results showed that the performance decreases when the weight is increasing. In the proposed scheme, the weight is dependent on the slotcount of current query round. Therefore, the simulation results demonstrated that performances of proposed scheme outperform the Gen-2 algorithm and is more stable than Gen-2 algorithm though the number of tags is small.

\section{REFERENCES}

[1] W. Chen, and G. Lin, "An Efficient Anti-Collision Method for tag Identification in a RFID System," IEICE Trans. Commun., vol.E89-B, no.12, pp.3386-3392, Dec. 2006.

[2] C. Wang, M. Daneshmand, and K. Sohraby, "Optimization of Tag Reading Performance in Generation-2 RFID Protocol," Computer Commun., vol.32, Issue 11, pp.1346-1352, July 2009.

[3] EPCglobal, "EPC Radio-Frequency Identity Protocols Class-1 Generation-2 UHF RFID Protocols for Communication at 860MHz-960MHz, Ver.1.2.0," EPCglobal Inc., Oct. 2008.

[4] ISO/IEC, "Information Technology - Radio Frequency Identification for Item Management - Part 6: Parameters for Air Interface Communication at 860-960 MHz, 19000-6," ISO/IEC, 2006.

[5] Auto-ID Center, " $860 \mathrm{MHz}-930 \mathrm{MHz}$ Class 0 Radio Frequency Identification Tags Protocol Specification Candidate Recommendation, Version 1.0.0," June 2003.

[6] I. Lim, "Performance Evaluation of Q-Algorithm with Tag Number Estimation Scheme," International Journal of MICS, vol.8, no.0, pp.45-50, Feb. 2010.

[7] I. Lim, "A Mechanism for Dynamic Allocation of Frame Size in RFID System," International Journal of MICS, vol.6, no.4, pp.364-369, Dec. 2008. 


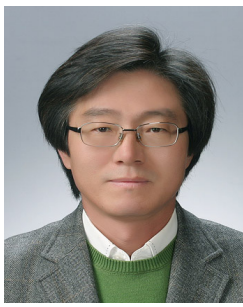

In-Taek Lim received the B.S. degree in computer science from University of Ulsan, Ulsan, Korea, in 1984, and the M.S. degree in computer science and statistics from Seoul National University, Seoul, Korea, in 1986. He received the $\mathrm{Ph}$. D. degree in computer engineering from University of Ulsan, Ulsan, Korea, in 1998. From 1986 to 1993 , he was a Senior Researcher at Samsung Electronics Co., Ltd. In 1998, he joined the faculty at Pusan University of Foreign Studies, Busan, Korea, where he is currently a Professor in the Department of Embedded IT. From July 2006 to June 2007, He was a visiting scholar at Cleveland State University in USA. His research interests include the MAC protocol design, ad-hoc network, RFID, and mobile computing. 